\title{
Non-Cancer Related Death
}

National Cancer Institute

\section{Source}

National Cancer Institute. Non-Cancer Related Death. NCI Thesaurus. Code C156428.

A death attributed to any cause other than the progression of a cancer-related

pathologic condition. 